\title{
11 Umgang mit weiblicher und männlicher Dissozialität am Jugendgericht im Kanton Bern
}

\author{
Dieter Hebeisen
}

Bei der komplikationslosen Geburt des Kindes ahnen die wenigsten Eltern, welche Ereignisse sie in der Pubertät und Adoleszenz ihrer Sprösslinge erwarten werden. Jeder Fortschritt des kleinen Wesens erfreut die stolzen Eltern, höchstens leicht getrübt durch schlaflose Nächte, weil der neue Erdenbürger mit seinem Schreien keine Rücksicht auf ein „menschliches Dauerbedürfnis“ nimmt. Strahlt das Kind am nächsten Tag seine Umgebung an, kann ihm keiner mehr böse sein und der Ärger wegen der schlaflosen Nacht ist bald einmal vergessen und vergeben. Nur ca. zwölf, dreizehn Jahre später sind aus den ehemals niedlichen Wesen zum Teil „rotzfreche“, unablässig nörgelnde und widerspenstige Halbwüchsige geworden, die nunmehr durch ihr ,aufmüpfiges“ Verhalten, absolut „chaotische“ Zimmer, schlechte Launen, überkritische Fragen, extreme Ansichten und vieles mehr auffallen, was die Eltern rasch an die Grenzen ihrer pädagogischen Leistungsfähigkeit zu bringen mag. Die ehemals so problemlosen Kinder halten sich nicht an Verbote oder verlangen stets eine Begründung, die sie nach ihren eigenen Maßstäben zu überzeugen hat - was von vornherein ein praktisch unmögliches Unterfangen ist, da selbst die objektive Begründung nicht akzeptiert werden kann. Plötzliche Weinkrämpfe oder Wutausbrüche machen Eltern völlig ratlos, der rapid absinkende Notendurchschnitt beschäftigt nicht nur die Familie, während der steigende Fernseh, Video- und Internetkonsum unweigerlich zu Geräteeng- 
11 Umgang mit weiblicher und männlicher Dissozialität am Jugendgericht im Kanton Bern

pässen führt. Das noch bis vor kurzem brave, wohlerzogene Kind stellt unverhofft unbequeme persönliche Fragen, kritisiert ständig, akzeptiert keine der oft unbeholfenen Erklärungs- bzw. Verteidigungsversuche der Erwachsenen und hält diesen genüsslich Widersprüchliches vor. Wichtiges wird nur noch mit Altergleichen besprochen, die Kommunikation beschränkt sich auf das Handy und was nur im Entferntesten nach Erwachsenen aussieht, wird mit Aggressionen bekämpft. Diese Zeit ist nicht nur für die Erwachsenen schwierig, die sich oft in ihrer Ratlosigkeit fragen, warum sie dieses Verhalten der Heranwachsenden tolerieren müssen oder verzweifelt nach den Gründen ihrer scheinbar falschen Erziehung suchen. Schwierig ist diese Zeit auch für die Jugendlichen selber, die in nicht anders reagieren können, weil sie in Unsicherheit über ihre bisherige Orientierung geraten. Durch die neuartigen erlebten Veränderungen, muss der heranwachsende junge Mensch lernen, wer er ist und wie er mit seiner neuen Rolle zurechtkommen kann. Nach der turbulenten Pubertät schließt sich übergreifend die nächste schwierige Phase der Adoleszenz an. Das Abschiednehmen von der Kindheit passiert bekanntlich nicht in einem Schritt. Während einer längeren Zeitperiode ist der junge Mensch nicht mehr Kind, aber auch noch nicht erwachsen. Das zeigt sich etwa darin, so werden einerseits die unangenehmen Seiten des Erwachsenwerdens - wie z.B. das Übernehmen von Pflichten oder das Einhalten von gültigen Vereinbarungen - oft weit in die Zukunft verschoben und andererseits die Rechte als Erwachsener beansprucht. Diese Zeit der Unsicherheit über sich selber, häufig gepaart mit Minderwertigkeitskomplexen, führt nicht selten zu Provokationen gegenüber den Erwachsenen. Ein Jugendlicher in der Adoleszenz kann seinen ehrgeizig joggenden Vater in seiner persönliche Lebenskrise zur Weißglut bringen, wenn er jedes Mal, wenn der väterliche Sportler verschwitzt und nach Luft ringend nach Hause kommt, genüsslich eine Zigarette anzündet und den Rauch möglichst tief inhaliert. Oder wenn die bewusst verschuldeten schlechten Noten in der Schule zu einer Krisensitzung nach der andern führen, bei welchen sich Lehrerschaft und Eltern verzweifelt bemühen, dem desolaten Zustand zu beheben, während der Halbwüchsige halb belustigt, halb gelangweilt, aber sicher immer passiv sich zurücklehnt und keinen eigenen Beitrag leistet. Dabei ist es dem scheinbar „coolen“ Jugendlichen meist alles andere als wohl in seiner Haut. Die neuen an ihn gestellten Anforderungen erzeugen Angst, verunsichern ihn und lassen erschweren ihm Hilfe von anderen anzunehmen. Diese Erscheinungen gehören mehr oder weniger ausgeprägt zum Erwachsenwerden und stellen eine schwierige Zeit sowohl für die Heranwachsenden wie auch für die Erziehenden dar, in der es nicht selten zu eigentlichen Zerreißproben kommen kann. Obwohl die Pubertät und Adoleszenz hohe Anforderungen an alle stellen, können beiden Entwicklungsstadien auf Basis eines vertrauensvollen Verhältnisses zwischen Eltern und den Heranwachsenden erfolgreich gemeistert werden. Selbst sehr schwierige und lang andauernde Pubertäts- bzw. Adoleszenzphasen dürfen nicht mit dissozialem Verhalten gleich gesetzt werden. 
Im „Kompendium der Psychiatrie und Psychotherapie“ wird der Terminus „Dissozialität“ als deskriptiver Oberbegriff für Störungen des Sozialverhaltens, Delinquenz und Verwahrlosung verwandt, wobei von den Störungen des Sozialverhaltens als kinder- und jugendpsychiatrische Entität die Delinquenz als juristisch-kriminalistisch manifest gewordenes abweichendes Verhalten und die Verwahrlosung als persistierendes Verstoßen gegen die sozialen Normen unterscheiden werden. Einfacher ausgedrückt und auf junge Menschen bzw. auf meine Arbeit als Jugendgerichtspräsident bezogen verstehe ich unter Dissozialität Folgendes:

- Missachten von sozialen Regeln und Normen

- Ablehnen von Verantwortung und Verpflichtungen

- Geringe Frustrationstoleranz und leichte Reizbarkeit

- Niedrige Gewaltschwelle, oft verbunden mit hohem Gewaltpotenzial

- Mangelhaftes oder fehlendes Schuldbewusstsein (keine Einsicht ins Unrecht der Tat)

- Unvermögen, Beziehungen einzugehen oder diese längerfristig aufrecht zu erhalten

- Rücksichtslosigkeit gegenüber andern, insbesondere auch gegenüber deren Meinungen und Gefühlen

Dabei steht fest, dass das Norm abweichende oder delinquente Verhalten deutlich, andauernd und eine gewisse Intensität aufweisen muss. Nur schwerwiegende stets gegen soziale Gesellschaftsnormen verstoßende Verhaltensweisen oder wiederkehrende Delinquenz mit mangelnder Schuldeinsichtsfähigkeit lassen den Schluss zu, dass Dissozialität vorliegen kann. Die Grenzziehung zwischen Norm abweichendem Verhalten, das auf die Pubertät oder Adoleszenz zurückzuführen ist und einem dissozial zu bezeichnenden Verhalten ist nicht leicht. Gerade bei Heranwachsenden, die aus ihrer inneren Unsicherheit heraus nicht immer adäquat handeln oder reagieren, ist Zurückhaltung angebracht, bevor man sie vorschnell als dissozial abstempelt. So kann ein notorischer „Töfflifrisierer“ (Mofafrisierer), der ganz klar gegen das Gesetz verstößt, ebenso wenig die Siebzehnjährige, die tagtäglich der Erwachsenenwelt überdeutlich zeigt, dass „sie sie alle mal können“, nicht als dissozial bezeichnet werden.

\subsection{Wie äußert sich Dissozialität in der täglichen Arbeit mit Jugendlichen am Jugendgericht?}

Vorab sei mit aller Deutlichkeit festgehalten, dass nur ein geringer Teil der Jugendlichen, mit denen sich die Jugendgerichte befassen müssen, weist dissoziale Auffälligkeiten auf. Es ist aber festzustellen, dass diese Zahl in den letzten Jahren überproportional zugenommen hat und vor allem deshalb gewichtet, weil die verantwortlichen sozialen Behörden an Grenzen stoßen. Die 
11 Umgang mit weiblicher und männlicher Dissozialität am Jugendgericht im Kanton Bern

konstante Ablehnung und Bekämpfung jeglicher pädagogischer oder therapeutischer Maßnahmen durch die betroffenen Jugendlichen erfordert von den Fachpersonen der Jugendhilfe viel Durchhaltevermögen und Fantasie. Und dennoch kann der Erfolg ausbleiben, wenn ein besonders hoher Dissozialitätsgrad vorliegt. Ob sich die Bemühungen der Jugendstrafrechtsbehörden zu einem späteren Zeitpunkt positiv auswirken, ist mangels entsprechender Untersuchungen nicht eindeutig zu beantworten, da die sich beharrlich widersetzenden Jugendlichen nach Ablauf der maximalen Frist der Maßnahme entlassen werden müssen. In dem entsprechenden Beschluss steht dann: „Die Maßnahme wird wegen Sinn- und Zwecklosigkeit per sofort aufgehoben.“ Diese vom Gesetzgeber nicht vorgesehene Variante verdeutlicht, dass einerseits diese Tendenz eine neuere Entwicklung darstellt und andererseits der betroffene junge Mensch zwar nach wie vor maßnahmebedürftig, aber nicht maßnahmefähig ist. Diese Tatsache ist nicht auf Anhieb erkennbar, sonst könnten die Justizbehörde im Vornherein auf das Anordnen von Maßnahmen verzichten. Viele Jugendliche weigern sich schon zu Beginn sich in einer pädagogischen oder therapeutischen Maßnahme zu integrieren, da dies mit dem Verlust von Freiheiten einhergeht und oft mit großen Ängsten verbunden ist. Die meisten jungen Menschen brauchen eine gewisse Zeit, bis sie sich an die neuen Strukturen und Vorgaben für sich akzeptieren können.

Die Justiz hat sich mit dissoziale Jugendlichen zu befassen hat, die dadurch auffallen, dass sie

- sich absolut lustlos verhalten (Nullbockstimmung)

- sich gegenüber allem und jedem verweigern

- ihren Überdruss an der Schule oder Ausbildung so ausgeprägt zeigen, dass es zum Ausschluss oder Abbruch der Ausbildung kommt

- in keinen Arbeitsprozess einzugliedern sind

- Drogen brauchen, um den Alltag zu bewältigen

- sich tage- bzw. nächtelang an ungeeigneten Orten aufhalten

v sich sämtlichen sozialen Strukturen entziehen

- sehr rasch äußerst aggressiv reagieren

- eine erhebliche Bereitschaft zu Gewalt zeigen

- sich Feindbilder aufbauen, um von sich selber abzulenken

- Fehler nie bei sich selber suchen

- nicht bereit sind, Verantwortung zu übernehmen

- unfähig sind, Beziehungen einzugehen bzw. über längere Zeit aufrecht zu erhalten, etc.

Auffallend ist, dass das Norm abweichende Sozialverhalten in den letzten Jahren wesentlich deutlicher zugenommen hat als die Delinquenz, obwohl mehr Fälle vorkommen, die in die persönliche Integrität der Mitmenschen eingreifen (Gewalttätigkeiten) festzustellen sind. Bezüglich der Delikte sind insofern enorme Unterschiede zwischen männlichen und weiblichen Jugendlichen festzustellen, als dass gemäß Anzeigestatistik das Verhältnis zwischen delin- 
quenten männlichen und weiblichen Jugendlichen zwischen 5:1 und sogar 10:1 liegt. Aus diesen Zahlen darf nicht fälschlicherweise der Schluss gezogen werden, Delinquenz - und damit auch Dissozialität - sei primär ein Problem der männlichen Jugend. Die Unterschiede in der Anzeigehäufigkeit haben andere Ursachen, z.B. dass weibliche Jugendliche männliche Gleichaltrige für sie die strafbaren Handlungen ausführen lassen, dass sich Straftäterinnen wesentlich geschickter und raffinierter anstellen und deshalb weniger schnell erwischt werden. Die männlich dominierte Polizei neigt zudem dazu, bei weiblichen Personen eher einmal „Gnade vor Recht“" walten lassen. Ebenso ist unzutreffend, dass weibliche Jugendliche nur so genannte leichte Delikte begehen. Gewaltdelikte, schwere Eigentumsdelikte, der Konsum harter Drogen etc. gehören heute auch zum Repertoire von delinquenten weiblichen Jugendlichen. Nicht zutreffend ist die Annahme, dass weibliche Jugendliche primär autoaggressiv sind. Ihre nach außen gerichteten Aggressionen sind in der Regel subtiler, können aber auch ganz brachiale Formen annehmen. Zusammengeschlagene Erzieherinnen und Erzieher, niedergemachte Beraubte, herausgerissene Sanitäreinrichtungen, zertrümmerte Möbel und abgefackelte Klinikstationen belegen dies.

Während bezüglich der Delinquenz primär ein quantitativer Unterschied zwischen Straftäterinnen und -tätern besteht, gibt es bezüglich Norm abweichendem Sozialverhalten eher qualitative Differenzen. Weibliche Jugendliche verstehen es besser, Vorteile des sozialen Umfeldes zu nutzen. Gerade Väter solidarisieren sich gerne mit ihren Töchtern, entschuldigen deren Fehlverhalten, decken sie mit Falschaussagen oder akzeptieren ihr Apathie. Weibliche Jugendliche setzen sich häufiger und bestimmter über Regeln und Abmachungen hinweg. Es werden mehr 12- bis 14-jährige weibliche als männliche Jugendliche in den frühen Morgenstunden aufgegriffen. Nicht selten ist die Antwort der Eltern „wir finden es auch nicht gut, dass unsere Tochter so spät unterwegs ist, aber was kann man dagegen machen? Sie nimmt sich dieses Recht einfach heraus und wir können sie ja nicht einsperren. "Im jugendstrafrechtlichen Verfahren setzen weibliche Jugendliche auffällig mehr Energie frei und nutzen alles zu ihrem Vorteil. Männliche Jugendliche zeigen eine größere Passivität, kämpfen schon in der Untersuchungsphase weniger gegen die Maßnahme, sondern verlegen ihre Aktivitäten in den Vollzug. Bei weiblichen Personen stehen in erster Linie psychische Auffälligkeiten oder alle Formen der Essstörungen im Vordergrund.

\subsection{Ursachen der Dissozialität}

Es stellt sich die Frage, welche Ursachen für die Zunahme dissozialer Jugendliche bestehen. Im Folgenden soll auf einige Aspekte etwas näher eingegangen werden. Vorab ist auf ein Erbe der 68er Bewegung hinzuweisen. 1963 erschien nach jahrzehntelanger Emigration Marcuse wieder auf der europäischen Sze- 
11 Umgang mit weiblicher und männlicher Dissozialität am Jugendgericht im Kanton Bern

ne, nachdem er in den USA bereits zum „most influential thinker“ des 20. Jahrhunderts und der „Neuen Linken“ aufgerückt war. Zusammen mit alten und neuen Weggefährten wurde er rasch zum geistigen Haupt einer weltweiten Bewegung, der sog. Frankfurter-Schule. Seine kämpferische Haltung richtete sich vorwiegend gegen das Feindbild einer Gesellschaft, wie er sie in Amerika glaubte erkannt zu haben, gegen das Schwinden einer Kultur als Folge einer immer zunehmenden Herrschaft technologischer Rationalität und gegen jene eines konsequenten Operationalismus. Dieses Gesellschaftssystem galt es nicht bloß zu negieren, sondern durch revolutionäres Handeln zu verändern, da es die Gewalt nicht grundsätzlich ausschloss. Die in diesem Gesellschaftssystem wirkenden Gesetzmäßigkeiten reproduzieren dauernd soziale Ungerechtigkeiten, zwischenmenschliche Entfremdung und totalitäre Macht. Das galt als Legitimation für das revolutionäre Handeln. Ein Fortschritt hin zur Humanität war daher nur durch eine radikale Opposition gegen den gesellschaftlichen Status quo möglich. In einer meines Erachtens genialen ideologischen Strategie hat die Frankfurter-Schule deshalb zwei maßgebliche Denksysteme miteinander verbunden, deren Schöpfer selbst kaum an eine solche Heirat gedacht hätten: Marx und Freud. Diese Verbindung lässt sich am besten am Beispiel der Begriffe repressiv und antiautoritär darlegen. Freud verwendete den Begriff „repressiv“ im Sinne von „verdrängen“. Marcuse gab diesem nun eine neue, gewaltige Ausdehnung, denn repressiv hieß nun „unterdrücken“. Damit schaffte er die Verbindung zu Marx, der ja mit dem Anspruch aufgetreten war, das jahrtausend alte Herrschaftsprinzip zwischen Unterdrückten und Unterdrückern aufzuheben. Marcuses zentrales Thema war ebenfalls die Ausbeutung der Beherrschten durch die Herrschenden. Repressiv im Sinn von unterdrücken war fortan die ganze Gesellschaft: die Eltern, die Familie, alle Institutionen, die Kirche, die Schule, die antiquierte Sexualordnung und vor allem auch die Rechtsprechung, welche nicht selten aus Tätern Opfer macht und dabei die eigentlichen Opfer vollständig vergisst. Nun wäre aber die Repression im Sinne von Unterdrückung nicht möglich, wenn der Mensch nicht vom Glauben an Autorität und an Autoritäten beseelt gewesen wäre. Der Kampf gegen die Unterdrückung musste sich daher gegen alles richten, was offen und unterschwellig für sich Autorität beanspruchte. Die ideologischen Strategen der „Neuen Linken“ wählten erneut ein raffiniertes Vorgehen, indem sie die politisch-ideologische Absicht in den Hintergrund stellten und dafür die antiautoritäre Haltung als extreme Form des pädagogischen Prinzips des „Wachsen-Lassens“ (anstelle von „Führen“) propagierten. Marcuses Unterfangen wurde gestützt durch die Tatsache, dass der Begriff autoritär schon immer einen leicht negativen Beigeschmack hatte. Die antiautoritäre Welle spülte alles weg, was wahrscheinlich zu Recht Autorität beanspruchen durfte. Das „Wachsen-Lassens“ wurde in der Folge so interpretiert, dass sich nur zum Guten entwickeln kann, was sich aus eigener Kraft und ohne äußere Einmischung entwickelt hat. Was nur schon von weitem nach Strukturen oder gar Vorschriften aussah, wurde abgelehnt und be- 
kämpft. Erst viele Jahre später wurde erkannt, dass den jungen Menschen kein Gefallen erwiesen wurde, sondern dass sie im Stich gelassen worden sind. Die gravierenden pädagogischen Spätfolgen wirken sich bis in die heutige Zeit aus.

Ein weiteres Element mit Einfluss auf das Auftreten der Dissozialität ist im „Egoismus“ zu finden. Gerade in sozialen Bereichen wurde die Forderung nach der individuellen Verwirklichung zum kategorischen Imperativ. Plötzlich wurde das Individuum nicht mehr als notwendiger Teil der Cemeinschaft gesehen, sondern aus dieser herausgelöst. Nur was das Individuum fördert, wurde als gut bewertet und der notwendige Beitrag als Teil des Ganzen wurde völlig negiert. „Verwirkliche dich selbst!“ wurde zum alles überragenden Credo. Diese Entwicklung ist nicht abgeschlossen und wird noch immer gefördert. Die Haltung ,ich und nochmals ich, und dann lange nichts mehr" sollte jedoch einem gemeinschaftlicheren Denken. Durch den extremen Egoismus wird verkannt, dass das Individuum längerfristig nicht ohne das Ganze überleben kann. Dieses aber wird durch den extremen Egoismus gerade zerstört.

Mit diesen Phänomenen steht ein Wertewandel zusammen. Evolution gehört selbstverständlich zu jedem lebenden und lebendigen System. Ein Wertewandel in Form einer Abkehr von wichtigen Normen insbesondere der Ethik steht hingegen nicht in Einklang mit einer sinnvollen Evolution. Als Beispiel wird auf die Darstellung des Menschen vor allem in den Medien und insbesondere auf die Möglichkeiten im Internet hingewiesen. Während sich die Fernsehsender durch die Anzahl von täglichen, immer brutaler werdenden Beiträge voyeuristisch ausgestalteten und sich banalen Sexsendungen zu überbieten suchen, widmen sich erschreckend viele Gefäße des Internets menschenverachtenden Darstellungen allertiefster Stufen.

In einer Welt, in der der Materialismus dominiert und Konsum oder Luxusgüter im Überfluss vorhanden sind, fällt es den Erziehungsverantwortlichen anscheinend schwerer, einfach einmal „Nein“ zu sagen. Die Verkaufsstrategien der Handelunternehmen fördern das Konsumverhalten und erschweren den Eltern die Erziehung. Schwierig wird es aber erst, wenn das dringend angebrachte „Nein“ nie kommt, bzw. gleich wieder aufgehoben wird, z.B. wenn die Eltern dem Kind zuerst etwas verbieten, auf dessen heftige und lautstarke Intervention dann aber doch erlauben, gekoppelt an das Versprechen, sich fortan zumindest ruhig zu verhalten. Diese Inkonsequenzen ortet schon das Kleinkind sehr rasch und kann effiziente Strategien entwickeln, die mit zunehmendem Alter immer ausgeklügelter und erfolgreicher werden. Dieses Nachgeben der Eltern ist nicht immer darauf zurückzuführen, dass sie ihr Kind nicht erziehen möchten, sondern eher darauf, dass sie erzieherisch verunsichert sind, da für sie viele Normen und Werte in Frage gestellt sind oder keine Gültigkeit mehr haben. Das Kind erhält in der Folge immer mehr Rechte und erlebt laufend seltener Grenzen. Diese scheinbaren Freiheiten führen in der Regel nicht dazu, dass der junge Mensch glücklicher ist. Seine Kompe- 
11 Umgang mit weiblicher und männlicher Dissozialität am Jugendgericht im Kanton Bern

tenz zu Eigenverantwortung wird nicht gestärkt und er ist oft orientierungslos. Sein Verhalten kann dazu führen, dass er sich im sozialen Rahmen unangemessen verhält und möglicherweise ausgegrenzt wird.

Nach dem ZGB gelten alle Menschen mit Ausnahme der Entmündigten ab dem 18. Altersjahr als erwachsen. In meiner täglichen Arbeit zeigt sich, dass diese gesetzlich klare Regelung nicht so eindeutig für die Betroffenen ist. Damit sind nicht in erster Linie 18-Jährige gemeint, die sich weigern, erwachsen zu werden und Verantwortung für sich zu übernehmen, sondern z.B. 30-Jährige, die ihr „Hotel Mama“ mit all seinen Vorteilen nie verlassen wollen. Noch ausgeprägter wird es, wenn offenbar sich ins Jugendalter zurücksehnende ältere Menschen mit verkehrt aufgesetzter Baseballmütze waghalsig durch die Gegend bladen und Sprachbrocken der 15-Jährigen vor sich hinmurmeln. Dies hat nichts damit zu tun, dass heute erfreulicherweise bis ins hohe Alter an der eigenen Fitness gearbeitet wird. Die 40-Jährige Mutter, die mit ihrer knapp 15-Jährigen Tochter wetteifert, um möglichst jung auszusehen, verkennt immer, dass sie verlieren muss, wenn sie einen derartigen Wettbewerb beginnt und Jugend zu ihrem Ideal stilisiert. Sie vergibt zudem all die Werte, die sie aufgrund ihrer Lebenserfahrung gerade von ihrer Tochter unterscheiden würde. Diese „Infantilisierung der Gesellschaft“ verwischt nicht nur die Grenzen zwischen Kindern und Erwachsenen, sondern wirkt sich negativ aus. Auf der einen Seite mischen sich Erwachsene in einen Bereich ein, den sie verlassen haben müssten und werden dadurch für die Jugendlichen zu einer echten Bedrohung. Auf der andern Seite wird das Alter herabgewürdigt, indem die ewige Jugend als alleine selig machend verkündet wird. Dem bejahrten Mitglied der Gesellschaft, das mit seiner reichhaltigen Erfahrung den nachkommenden Generationen Wesentliches mitgeben könnte, bleibt weder Raum noch Zeit. Damit wird ein vielfältiges und wichtiges Potenzial intergenerationaler Interaktion nicht angemessen nutzbar.

Wie sollen Kinder und Jugendliche beziehungsfähig werden, wenn das sie umgebende soziale Umfeld ihnen nicht die Möglichkeit bietet, Beziehungen eingehen zu können? Auch wenn Scheidungen bei 40\% der geschlossenen Ehen vorkommen, können geschiedene Eltern oder neu zusammengesetzte Partnerschaften sehr wohl einen fruchtbaren Boden für die gesunde Entwicklung von Kindern und Jugendlichen darstellen. Problematisch sind familiäre Umstände, die den Jüngsten der Gesellschaft äußerst schlechte Startbedingungen schaffen, z.B. Kinder von suchtabhängigen oder HIV-positiven Müttern; Kinder, um die im Rahmen der Scheidung gestritten oder gar gekämpft wird; unerwünschte Kinder; Kinder, die als Folge einer Vergewaltigung geboren wurden; Kinder, die aus fremden Kulturen herausgerissen werden etc. Die schlechten Startbedingungen können in der Regel kaum mehr kompensiert werden. Die Folgen von Frühverwahrlosungen können zwar während einiger Zeit unter optimalen Bedingungen aufgefangen werden, brechen aber in der Regel mit aller Vehemenz spätestens in der Pubertät voll durch. Nicht selten wiederholt sich dann von Generation zu Generation dasselbe Schicksal. 
Eine weitere mögliche Ursache von Dissozialität bei Jugendlichen steht mit sexuellem Missbrauch im Zusammenhang. Gerade bei weiblichen Jugendlichen, die in einem stationären Rahmen untergebracht werden müssen, zeigt sich eine hohe Zahl von Fällen, bei denen Missbrauch vermutet wird. Da die häufigsten sexuellen Übergriffe im unmittelbaren sozialen Umfeld stattfinden, bleibt vermutlich in Zukunft die Dunkelziffer noch hoch. Die Auswirkungen auf die jungen Menschen beiderlei Geschlechts sind prägend für das ganze Leben.

\subsection{Besteht somit nun Grund zur totalen Resignation?}

Es trifft zu, dass bei schweren Störungen im Sozialverhalten die zuständigen Behörden an die Grenzen des Machbaren gelangen und resignieren müssten. Diese dissozialen Jugendlichen überfordern das Hilfesystem, jedoch ist die Zahl dieser schwierigen Fälle niedrig. Es muss davor gewarnt werden, dass jedem Fall Norm abweichenden Verhaltens auf Dissozialität zu schließen. Dennoch darf eine weitere zunehmende Entwicklung nicht unbeachtet bleiben.

Wie ist deshalb dieserTendenz zu begegnen, denn die Ursachen der Dissozialität sind verschieden und kombiniert. Es ist bei den Ursachen anzusetzen, um vor allem in der Anfangsphase des sich abzeichnenden dissozialen Verhaltens mit pädagogischen und therapeutischen Maßnahmen viel zu bewirken. Wichtig dürfte dabei sein, dass nicht zu schnell resigniert wird, auch wenn die Erfolgsaussichten nicht nur im ersten Moment, sondern für längere Zeit gering erscheinen. Die zuständigen Fachpersonen und der junge Mensch selbst sind bei der Behandlung stark gefordert, um Eigenverantwortung und positiven Prozess einzuleiten.

Grundsätzlich müssen die Bemühungen über den Einzelfall hinausgehen. Die negativen Ursachen sind anzupacken. Dies wiederum erfordert ein Umdenken der Gesellschaft, sonst läuft der Sozialstaat Gefahr, dass durch ihn dissoziale Jugendliche ausgrenzt werden, wenn sie materiell gesichert, aber nicht integriert werden können.

Nachdem die letzten Jahrzehnte geprägt vom technischen Fortschritt waren, ist es an der Zeit, dass wir uns wiederum vermehrt dem Menschen zuwenden. In Lessings Schrift „Die Erziehung des Menschengeschlechts“ führt der Weg des Menschen zum Menschen über drei Stufen:

v von der Stufe der zehn Gebote - auf welcher Forderungen mit kategorischem Imperativ gestellt werden: du sollst, ... sonst ... -

- über die Stufe der Bergpredigt - auf welcher ohne Strafandrohung nur noch an die Einsicht des Menschen appelliert wird -

- zur höchsten Stufe hinauf, auf welcher der Mensch einzig um des Guten willen handelt, ohne Strafandrohung und ohne Aussicht auf eine Belohnung. 
11 Umgang mit weiblicher und männlicher Dissozialität am Jugendgericht im Kanton Bern

Die unteren Stufen sind notwendige Durchgangsstufen, die nicht übersprungen werden dürfen und die nie aus der innern Entwicklung des Menschen verschwinden werden. Die jeweils höhere Stufe überhöht die untere und nimmt ihr dadurch ihre Allmacht und Ausschließlichkeit. Erst auf der dritten Stufe - dem Handeln um des Guten willen - ist der Mensch „Mensch im eigentlichen Sinne“. Auch Pestalozzi geht in seiner gedankenschweren Schrift „Über den Gang der Natur in der Entwicklung des Menschengeschlechts“im Wesentlichen von einem dreistufigen Entwicklungsgang aus, den die Menschheit und der einzelne Mensch zu gehen hat. Nach Pestalozzi kann nur, wer diesen Gang kennt, in fruchtbarer Weise etwas zur Entwicklung des Menschen und der Menschheit beitragen. In seinem tierischen Zustand - dem Naturzustand, der an sich weder gut noch böse ist - folgt der Mensch seinem Instinkt; als Bürger im gesellschaftlichen Zustand - in welchem er am am meisten gefährdet ist - dem Gesetz; als Mensch im sittlichen Zustand gehorcht er hingegen seinem Gewissen. Erst im dritten Zustand kann von ihm als „Mensch im eigentlichen Sinn“ gesprochen werden. Ich bin überzeugt, dass gerade die heutige Zeit sich auf diesen Menschen im eigentlichen Sinn zurück besinnen muss. Dies hat nichts mit falscher Nostalgie oder Angst vor der Moderne zu tun. Die Zeit, in welcher eine unwahrscheinliche Entwicklung der Naturwissenschaften stattfindet, ist mehr denn je auf die Gültigkeit von ethischen und pädagogischen Normen angewiesen. Sonst besteht die Gefahr, dass Brechts voller Bitterkeit und Resignation in seiner Schrift „Galileo Galilei“ geschriebenen Worte eines Tages doch zutreffen: „Ihr Wissenschafter mögt mit der Zeit alles erkennen, was es zu erkennen gibt und Euer Fortschritt wird doch nur ein Fortschreiten von der Menschheit weg sein. Die Kluft zwischen Euch und ihr kann eines Tages so groß werden, dass Euer Jubelschrei über irgendeine neue Errungenschaft von einem universalen Entsetzensschrei beantwortet werden könnte.“

Es ist die Aufgabe aller, das Erbe von Sokrates fortzuführen und das Denken, Wissen und Erkennen im sittlichen Gewissen zu verankern, um die Ansprüche der Jugend und der nachfolgenden Generationen zu sicher zu stellen. 